\title{
Selection of Master Device in Wireless Personal Area Networks and its emerging services
}

\author{
Jahangir khan \\ School of computer science \\ PAF-KIET, Pakistan Air Force Base \\ Korangi Creek Karachi 75190 \\ Pakistan
}

\author{
Khurram shahid \\ School of computer science \\ PAF-KIET, Pakistan Air Force Base \\ Korangi Creek Karachi 75190 \\ Pakistan
}

\author{
Khisro khan \\ Directorate of Science and \\ Technology \\ TF 250, Deans Trade center \\ sadder cant, Peshawar k.p.k
}

Pakistan

\begin{abstract}
A wireless personal area network (WPAN), which is virtually a synonym since almost any personal area network would need to function wirelessly. Working on WPAN networks will enable us to gather advance knowledge about ongoing research on WPAN networks and invoke us to contribute new ideas towards WPAN and its emerging services. The main focus of this research is to investigate the different factors which are important for the formation of WPAN. we will propose and investigate novel handover algorithm that causes handover of the Master Device quickly and efficiently in Wireless Personal Area Network. Handover process, which needs to be fast and efficient for it not to have an effect on the mobile user's ongoing communication, occurs when a mobile user migrates to a different channel or to an adjacent cell. The proposed handover algorithm will be presented, modeled and simulated in this paper. WPAN standard have defined two standard handover algorithms based on Reach ability and Stability for the handover of master device in WPAN. These two techniques will be combined and simulated and compared with the battery power selection technique by OPNET modeler. Simulation results presented in this paper is to prove that the initiation of a PAN can be started with a TM, which could readily handover to the acquired PAN node which meets the master device (MD) selection criteria.
\end{abstract}

\section{General Terms}

Hand over process, master device, Reach ability, stability.

\section{Keywords}

Wireless Personal area network, MD, Performance comparison, OPNET modeler.

\section{INTRODUCTION}

The concept of a PAN first was developed by Thomas Zimmerman and other researchers at M.I.T's Media Lab and later supported by IBM's Almaden research lab. In a research paper, Zimmerman explains why the concept might be useful: As electronic devices become smaller, lower in power requirements, and less expensive, we have begun to adorn our bodies with personal information and communication appliances. Such devices include cellular phones, personal digital assistants (PDAs), pocket video games, and pagers. Currently there is no method for these devices to share data. Networking these devices can reduce functional I/O redundancies and allow new conveniences and services [11]. Working on WPAN networks will enable us to gather advance knowledge about on going research on WPAN networks and invoke us to contribute new ideas towards WPAN and its emerging services. The main focus of this thesis is to investigate the different factors which are important for the formation of WPAN.

A personal area network (PAN) is the interconnection of information technology devices within the range of an individual person, typically within a range of 10 meters. For example, a person traveling with a laptop, a personal digital assistant (PDA), and a portable printer could interconnect them without having to plug anything in, using some form of wireless technology. Typically, this kind of personal area network could also be interconnected without wires to the Internet or other networks. A wireless personal area network (WPAN), which virtually, a synonym since almost any personal area network would need to function wirelessly. Conceptually, the difference between a PAN and a wireless LAN is that the former tends to be centered around one person while the latter is a local area network (LAN) that is connected without wires and serving multiple users. In another usage, a personal area network (PAN) is a technology that could enable wearable computer devices to communicate with other nearby computers and exchange digital information using the electrical conductivity of the human body as a data network.

A wireless WPAN consists of a dynamic group of less than 255 devices that communicate within about a 33-foot range. Unlike with wireless LANs, only devices within this limited area typically participate in the network, and no online connection with external devices is defined. One device is selected to assume the role of the controller during wireless PAN initialization, and this controller device mediates communication within the WPAN. The controller broadcasts a beacon that lets all devices synchronize with each other and allocates time slots for the devices. Each device attempts to join the wireless PAN by requesting a time slot from the controller. The controller authenticates the devices and assigns time slots for each device to transmit data. The data may be sent to the entire wireless PAN using the wireless PAN destination address, or it may be directed to a particular device. The term Adhoc networks are often characterized by a dynamic topology due to the fact that nodes change their physical location by moving around. This favors routing protocols that dynamically discover routes over conventional routing algorithms like distant vector and link state. Another characteristic is that host/node has very limited CPU capacity, storage capacity, battery power and bandwidth, also referred to as a "thin client". This means that the power usage must be limited thus leading to 
a limited transmitter range. The access media, the radio environment, also has special characteristics that must be considered when designing protocols for ad-hoc networks. One example of this may be unidirectional links. These links arise when for example two nodes have different strength on their transmitters, allowing only one of the hosts to hear the other, but can also arise from disturbances from the surroundings. Multihop in a radio environment may result in an overall transmit capacity gain and power gain, due to the squared relation between coverage and required output power. By using multi hop, nodes can transmit the packets with a much lower output power. Working with a WPAN requires having a master-slave relationship to buildup the WPAN. Whereas when we consider ad-hoc networks, they require only peer-to-peer relationship between two or more devices to create a network to share resources. This gives rise to a new problem domain as far as the selection of master device is considered. So when two or more WPANs communicate with each other to establish a larger PAN in an ad-hoc fashion, it fails to respond to dynamic devices to select any of them as a master device because currently there is no scheme to cater this situation. This paper investigates the master device selection performance based on two main criteria which are Reach ability \& Stability and comparing the results with standard battery power selection algorithm.

Existing schemes for the selection of Master Device in a PAN encounter problems of ping-pong effect and network instability when two or more PANs enter the same boundaries to establish a larger PAN in an ad-hoc fashion. To overcome on the problem we have proposed a novel algorithm to select the Master Device in a Personal Area Network, communicating with other PANs in an ad-hoc manner, by considering Reach ability \& Stability as the two prime selection criteria along with other factors to solve the problems of ping pong effect \& network instability.

The paper proceeds as in pattern of sections: section 2 briefs for the approaches. Section 3 describes related work. Section 4 proceeds for the model and simulation scenario. $2^{\text {nd }}$ last describes results and last one section concludes the paper.

\section{APPROACH}

In this paper, we are trying to solve is that how to select master device when PAN acts as an ad-hoc nature and to solve this new network environment, we consider the previous two schemes (Stability \& Reach ability) as primary selection algorithms for a master device. Furthermore we have proposed a novel algorithm by focusing with new network dimensions and system boundaries. Our proposed novel algorithm acts efficiently when ever two or more PAN network interact in an ad-hoc basis by means of using relay/salve device which links two or more PANs or by PANs itself merge for an temporary/permanent basis, either of the situation, in both scenarios, 3 meters/second mobility was enabled and maximum PAN vicinity was assumed to have a range of 10 meters. Our proposed novel algorithm will demonstrate stable and eliminate the ping pong effect created by other algorithms if used under such scenario. Hence we can conclude that the both factors Reach ability and Stability are very important in selecting the master device.

\section{RELATED WORK}

This research based study may be categorized into different broad sub-domains on basis of the issues identified. The literature or the research papers which have been cited during this research study is given under here, spread over the following domains:

\subsection{Issues in Personal Area Networks}

In this section we will discuss the major issues and challenges that need to be considered when a PAN system is to be designed. The major factors that affect the design, deployment, and performance of a WPAN system are as follows:

\subsubsection{Routing}

The main responsibility of a routing protocol include exchanging the route information, finding the feasible path to a destination based on criteria such as hop length, minimum power required, and lifetime of wireless link, gathering information the path breaks, mending the broken paths expending minimum processing power and bandwidth and utilizing the minimum bandwidth. In PAN topology, some unpredictable situations with PAN devises such as sudden power-off or fast movement agitate PAN topology. To solve the mention problem the paper proposed a new SCS (Seamless Coordinator Switching) scheme [12] to maintain the pieonet by selecting a coordinator in the subsequent super frame without incurring the re- initialization overhead. Furthermore according to IEEE 802.15.3b standard the use of CTA (Channel Time Allocation) scheduling scheme which is not flexible for variable traffic thus creates delay, jitter and also consuming valuable "air-time" with signaling overheads. The author presented a new SCS (Seamless Coordinator Switching) [10], design method with selfreconfiguring topology. The PAN is self organized using alternative coordinator (as a successor) under unpredictable situation is provided by information in capability field in IEEE 802.15 WPAN MAC protocol. For preparing the unpredictable situation, the current master device broadcasts that order in every deacon frame. This particular piece of information is used for selection the alterative coordination under unexpected situation. The main objective of SCA scheme is to minimize the overhead association with possible re-initialization processes.

Further more in IEEE 802.15.4 for LR-WPAN (Low Rate Wireless Personal Area Network) it do not provide proper message scheduling algorithm for real time message transportation. The proposed solution is a message scheduling algorithm [4], which is suitable for scheduling a given periodic real time message set in the IEEE 802.15.4 (LRWPAN standard). Proposed Scheduling algorithm is based on measuring the time stamps of the GTS packets and comparing them with the calculated ones. The implementation result of the guaranteed time communication system which follows the schedule generated by the proposed algorithm on an IEEE 802.15.4 is accommodate with prototype board of packet sniffer of Chipcon. The proposed off-line message scheduling algorithm which is based on a distance constrained scheduler generates the standard specific parameters.

\subsubsection{Multicasting}

Multicasting plays an important role in main applications of ad hoc wireless networks, namely emergency search and rescue operations and military communication. In such environments, nodes from groups carry different tasks that require point to multipoint and multipoint-to-multipoint voice and data communications. The major issues in designing multicast routing protocols $[1,3,6,7]$ are as follows:

Robustness

Efficiency 
Control overhead

Quality of service

Efficient group management

Scalability

Security

The physical distribution and the dynamically changing conFigure ration of terminal devices in the PAN, makes PAN environment instable. The solution presented MAIPAN (Middleware for Application Interconnection in Personal Area Networks) architecture [3] which offers uniform application programming interface (API) to control the dynamically changing configuration of the PAN devices. By using MAIPAN architecture the PAN protocol is divided into 2 layers. The data layer aim to provide effective and secure data transport between applications. The control plane is responsible for managing pins, channels, sessions and for handling security and access control. MAIPAN is based on three concepts: pins, channels an sessions. Applications offering the services have input and output outlets, which are called pins-borrowing the expression from the integrated circuit world. Pins are the connection points of the applications to the middleware, so the middleware sees the applications in the PAN as a set of input and output pins. A pin has a pre-defined type, which shows the type of data that the pin can emit or absorb, that is, the type of information the application can handle (e.g., mouse movements, keystrokes). According to the needs new types can be defined any time. To enable communication between pins, the middleware creates and configuring channels, which are point-to-point links that interconnect pins.

\subsubsection{Self organization}

In the short-range WPAN environment, message propagation by flooding causing frames collisions which affect the performance of network. The presented solution is a novel effective scheme of configuring WPANs called Master-driven Connection Control (MaCC) [5], which avoid excessive message propagation by flooding causing frame collisions in the WPANs. The presented design goal of $\mathrm{MaCC}$ is to discover the shortest path without flooding with less amount of traffic load than flooding, by utilizing a characteristic of master-driven operations in WPAN. MaCC has three phases: first, the Parent Child Relationship-net (PCR-net) formation, second, the route discovery, and third, the connection establishment. (MaCC supports routing to discover the minimum hop-count routes without transmitting excessive queries or any nodes transmitting messages simultaneously. It also forms an optimal topology corresponding to the discovered routes dynamically. The results show control messages propagated in a MaCC network at route discovery are reduced to less than one half of those in a network utilizing pure flooding.

\subsubsection{Security}

Security for the communication is very important in ad hoc wireless network, especially in military applications. There are two kinds of attacks in ad hoc wireless networks, generally classified into two types:

Passive attacks-passive attacks usually refer to the attempts made by malicious nodes to perceive the nature of activities. To stop them pre-act mechanism [2] is used to stop malicious nodes activities by enabling checklist method.
Active attacks: active attacks disrupt the operation of the network. The attacks that are performed outside the network are called external attacks.

\subsubsection{Ping Pong Effect}

In PAN environments, the inefficiency of power management selection of Master device creates the ping pong effort which disturbs the PAN topology. To solve the mentioned problem the author suggests a self-reconfiguring topology is proposed to manage the mobility and recursively update the network topology. They also modify the mobility management scheme with fuzzy logic selection method to overcome the ping-pong effect. A selfreconfiguring topology [9], is proposed to manage the mobility and recursively update the network topology. This simple idea will follow towards enabling master device selection process to respond to the needs of the nomadic computer users.

\subsection{Master device selection criteria in PAN}

Existing schemes for the Selection of Master Device in PAN have many conflicts related to their work scope and system boundaries, as we move forward to more complex and multi-adhoc network environments, many problems arise when two or more ad-hoc networks enter same boundaries to establish a larger PAN network forcing to chose Single Master Device to Manage the PAN network entities. In such situation need to be solve to validate the Master Device among different Master Device Candidates. According to different schemes and methodologies, the main criteria for selecting a Master Device are Bandwidth, Processing power, Battery Power, Stability, Reach ability.

\subsection{Model of Reach ability and Stability}

Following are the details working models and concepts of Reach ability and Stability which are used in our thesis model.

\subsubsection{Reach ability Concept}

Reach ability in Personal Area Network means range in which all devices, which are in PAN, are directly reachable by the Master Device. There are different techniques to indicate whether a device is reachable. In one such technique when a device enters PAN it will broadcasts the beacon every 5 seconds, if master device receives the beacon it means that the device is within the range. Reach ability factor introduce node detection and internetworking mechanism between different PANs, whereas it also provide information exchange between different wireless networks by send beacon message to allow them to enter into their network. By using Reach ability element for the master device selection will give edge to the PAN where it can able to act in an ad-hoc fashion to work in mobile dynamic environment.

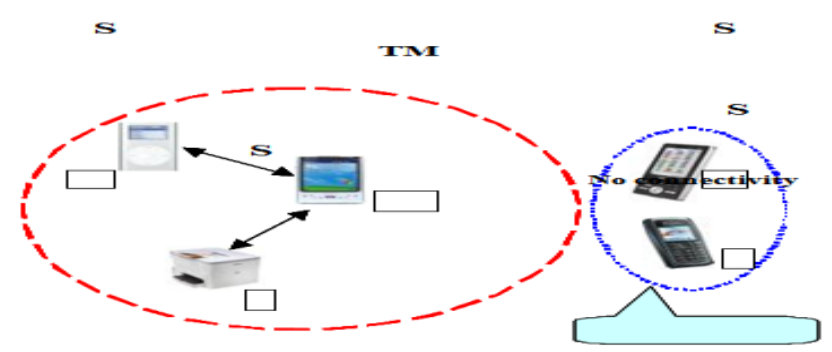

Figure 1. Scenario of Reach ability in PAN without connectivity 
The figure 1 . we can see that there are two circles, the red circle shows that there are three devices in a PAN, connected to each other with a Temporary Master Device(TM) is connected to two Slave devices (SD). The vicinity of PAN is only 10 meters. In this scenario we are using short distance wireless medium like WLAN with very low amount of power such as 7.4 e- 8 watts. The blue circles shows only two mobile devices connected to each other, but have no connectivity with the red circle as they are not within the vicinity of PAN. The two mobile devices in red circle can communicate between themselves on ad hoc basis, but would not be able to communicate with the devices in PAN as no device is present between then, which can act as a medium or source of communication between the red and blue circle.

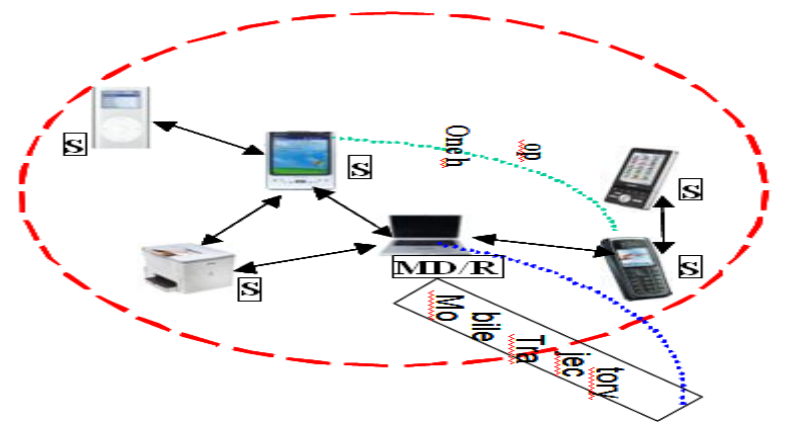

Figure 2. Shows a mobile node enables extension in PAN with its Reach ability and become MD

The red circle shows the vicinity of PAN for TM, as the two slave devices are out of the vicinity of TM. In simulation we enabled a mobile node which joins the PAN. Firstly, this node acts as slave device. In mean while, it is seen that this mobile node has a capability to reach the two devices outside the vicinity of last TM setup access. TM handovers the mastership to the incoming node coz of its reach ability feature to maximum number of nodes. New master device has maximum reach ability in the network as shown in Figure 2.

\subsubsection{Stability Concept}

Stability is list of all the devices within a PAN, which were connected to the Master Device, We can define stability as the history of all the devices joining and leaving the PAN. Stability is considered as one of the main factors for selecting the master device in PAN as it covers the reach ability and history of all other devices in PAN and updates the Master Device about them. We have divided the stability into the following grades for Master selection.

$\begin{array}{ll}\text { High } & >80 \mathrm{sec} \\ \text { Medium } & >=50 \mathrm{sec} \\ \text { Low } & <30 \mathrm{sec}\end{array}$

High- The devices which stay in PAN for more than 80 seconds are considered to be reliable and have better chance to act as master device.

Medium- The devices which stay in PAN for 50 seconds are considered to be not very reliable because 50 seconds as being part of PAN is not sufficient, so such devices do not have great chance of becoming the master device.

Low- The devices which stay in PAN for less than 30 seconds have minimum chance of becoming the master device, as they are considered totally unreliable.

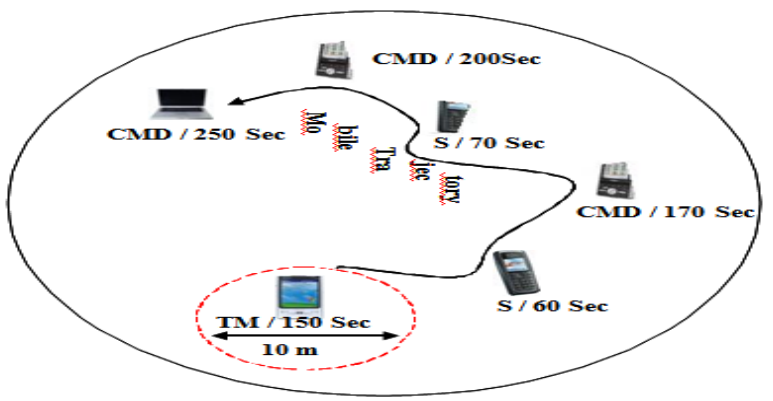

Figure 3. Shows scenario of Stability in PAN with candidate master devices during mobility

The scenarios in Figure 3. Show the stability criteria in PAN. We can see different mobile devices in the PAN vicinity with the time indicating they are part of PAN. The simulations have been carried the TM initiates the PAN as the most stable device in network and stay master throughout the simulation.

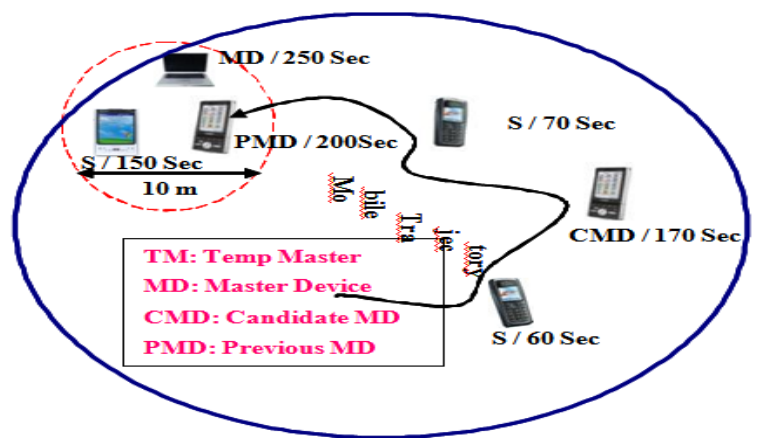

Figure 4. During mobile trajectory TM handovers to CMD and the device with highest stability time become MD

In Figure 4. we can see that TM initiates the PAN but during mobility, different nodes joined the PAN and their stability time is higher than the TM. In such case TM become a slave and the more stable node becomes new Master of the PAN. This continues till end of mobility as long as it does not find higher stable time period for another node in PAN such as candidate master device CMDs with different stability times. So the highest probability to become master device is a function of highest stability time.

\section{WPAN MODEL \& SIMULATION SCENARIO}

\subsection{Model Structure}

In the proposed model, we have configured three independent Pans which have different numbers of nodes communicating with each other. The system environment is configured according to standard mobile devices such as Laptops, PDAs, and Mobile Phones. By using existing tools available in OPNET 8.1 Version, we have successfully constructed the mention scenarios, further more we have also introduces new communication interfaces to make more scenario more realistic. This thesis is based on the performance comparison of existing algorithm. 


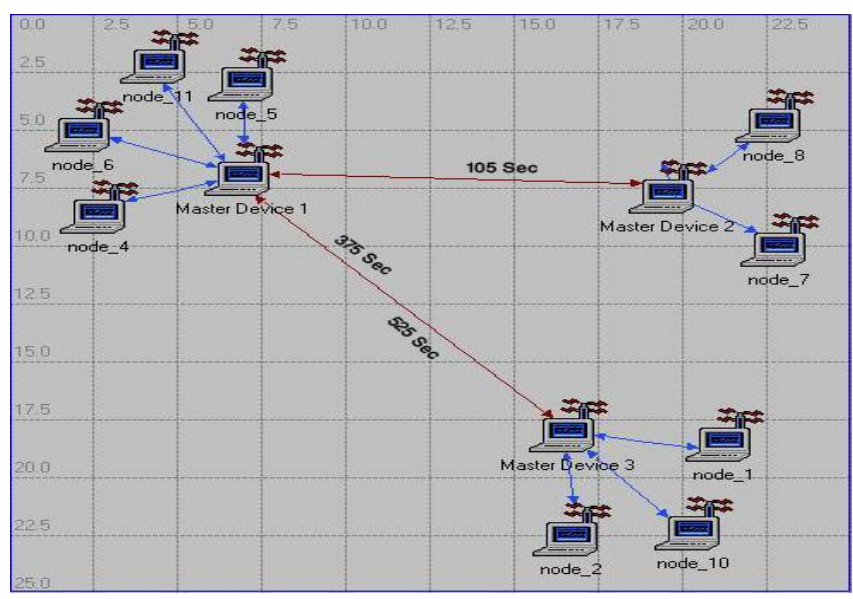

Figure 5. Model Scenario simulated

\section{Node architecture in OPNET}

Node models of some commonly used Wireless LAN objects in Opnet v11.1 are as follows:

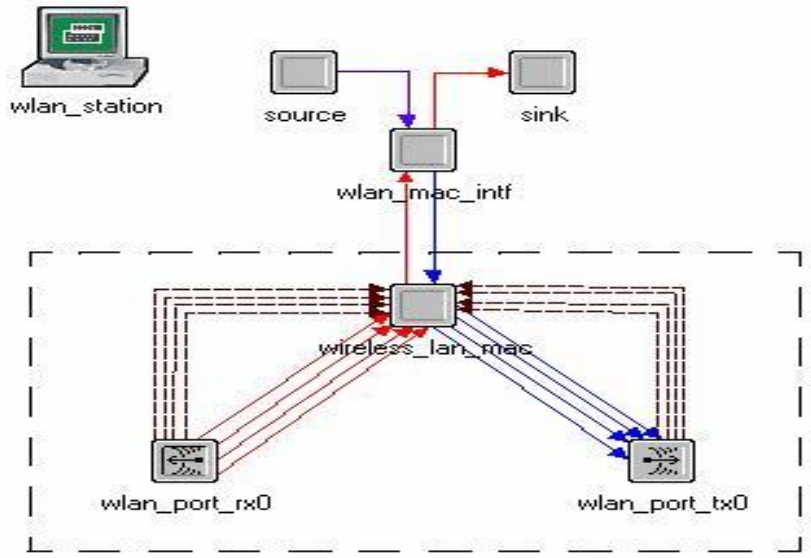

Figure 6. Architecture of Wireless station in Opnet v11.1

The Wireless PAN modeling of the MAC and the physical layer is comprised of the wireless lan_mac process, transmitter, receiver, and the channel streams (as shown by the box in Figure 6. The ARP (Address Resolution Protocol) is an interface between the MAC and the higher layers. To study the WPAN MAC without a higher-layer stack (such as TCP/IP and applications), use the station node model that uses source and sink models to simulate higher layers. The higher layers are replaced by a source and a sink process. There is a MAC interface that is equivalent to the ARP in the client/server model. The WPAN MAC process is independent of the higher layer as long as it has an appropriate interface. The IEEE $802.11 \mathrm{~b}$ standard is an amendment to 802.11 that adds support for a high-speed physical layer (PHY) extension in the $2.4 \mathrm{GHz}$ band.

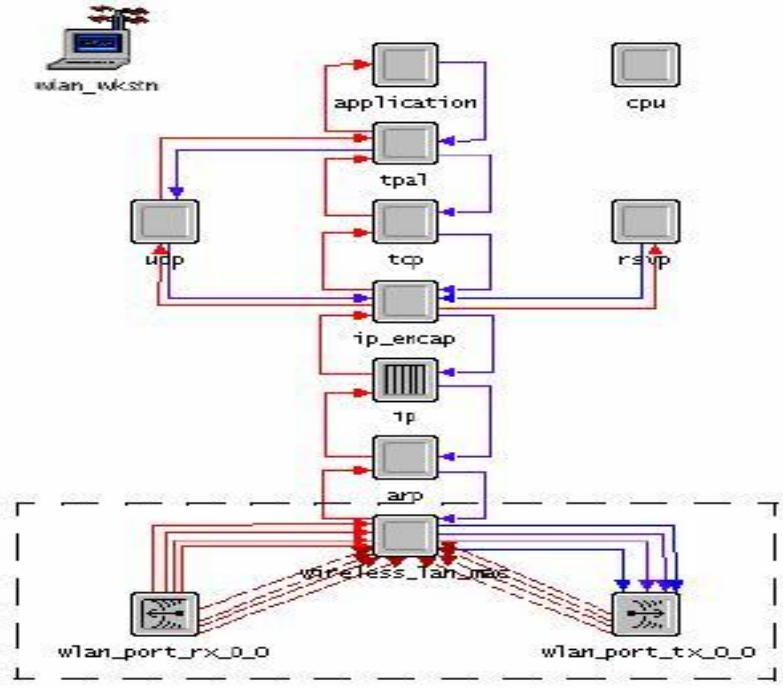

Figure 7. Architecture of wireless LAN workstation

The node model can be used to do the following: Generate controlled traffic in the WPAN network and evaluate the performance of MAC, Simulate the effect of WPAN attributes independent of the higher layer, Obtain shorter simulation time for large networks

The node model has the following station limitations: Bridges can be used to connect the BSSs of WPAN stations. A station can act as an access point, but it will function only as a relay within the BSS. We cannot observe the impact of MAC on the higher layer WPAN link layer additives.

\subsection{Our defined model layer in PAN}

For the master device selection, we will describe the function of link_layer which we have created in WLAN node in Opnet Modeler v10.0. In Figure 8. We can see an added model layer in the WPAN node model, this layer is above the link layer and we will discuss about it in detail later on.

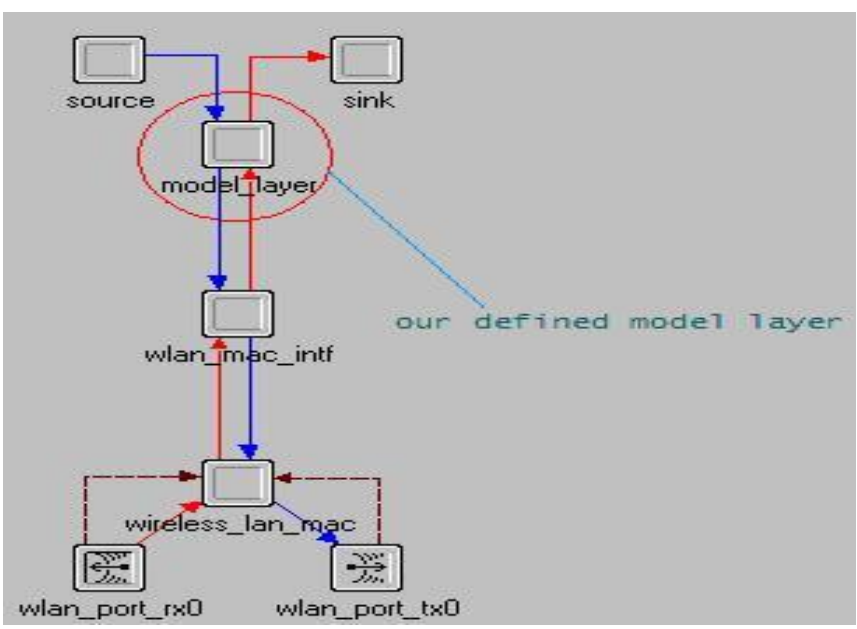

Figure 8. Our defined model layer in WPAN 


\section{RESULTS}

This section entirely deals with the simulation carried out in OPNET 8.1 to evaluate the performance of proposed master device selection algorithm using standard power selection method and proposed stability-reach ability algorithm.

\subsection{WPAN Battery Power Selection Results}

Figure 9. shows the simulation for model scenario as. In this scenario there are three different WPAN, which have no connection with each other. This simulation will execute 5 steps, in step1 the MD2 will interact with MD1 after 105sec, in step2 the MD1 and MD2 will undergoes master device selection according to batter power selection criteria, in step3 MD3 will interact with MD1 and in step4 MD3 will join MD1 after $375 \mathrm{sec}$ and in step5 MD3 leave MD1 after.

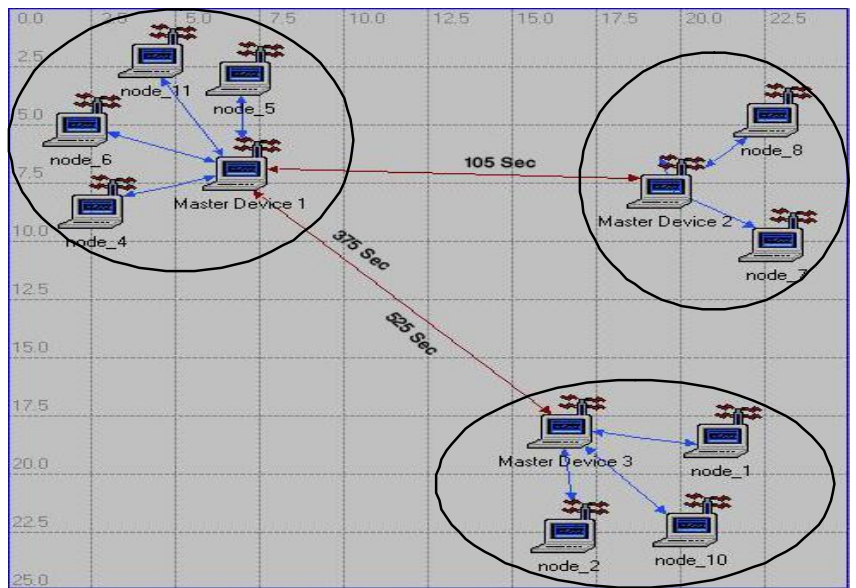

Figure 9. Scenario without connectivity between WPANs

\section{Step-1}

WPAN1 and WPAN2 are within vicinity of each other and now the both master device will communicate to select the primary master device.

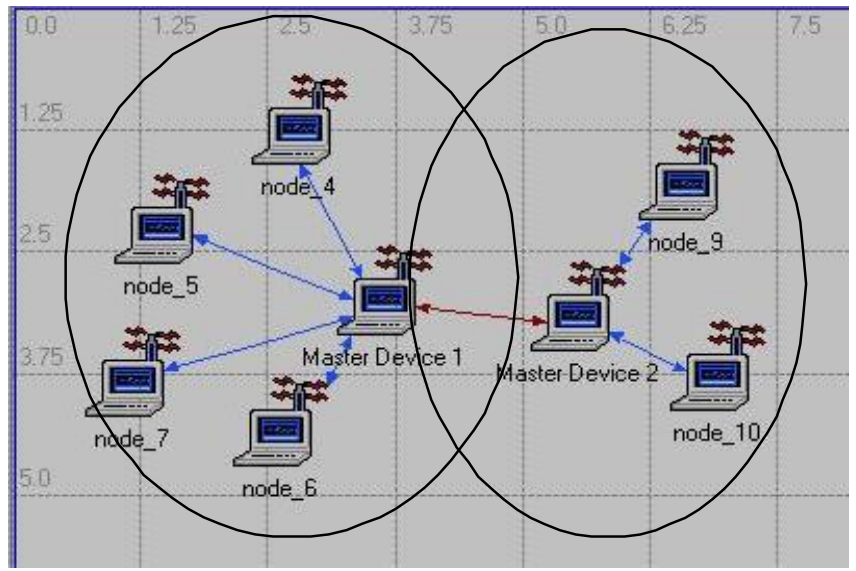

Figure 10. WPAN1 and WPAN2 interconnecting.

\section{Step-2}

MD1 handover all its attached nodes to MD2 and acts as candidate master device because the MD2 has more battery

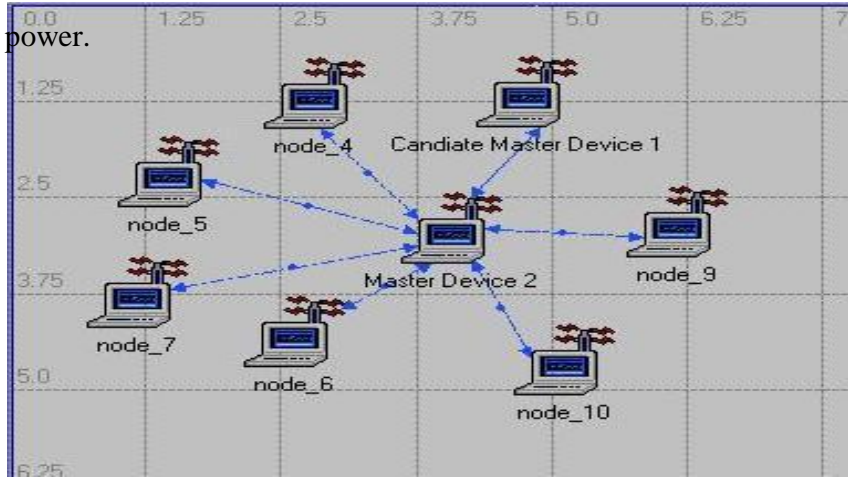

Figure 11. COMBINE WPAN

Step-3

Due to sudden loss of battery power of MD2, the primary master device handover to MD1.

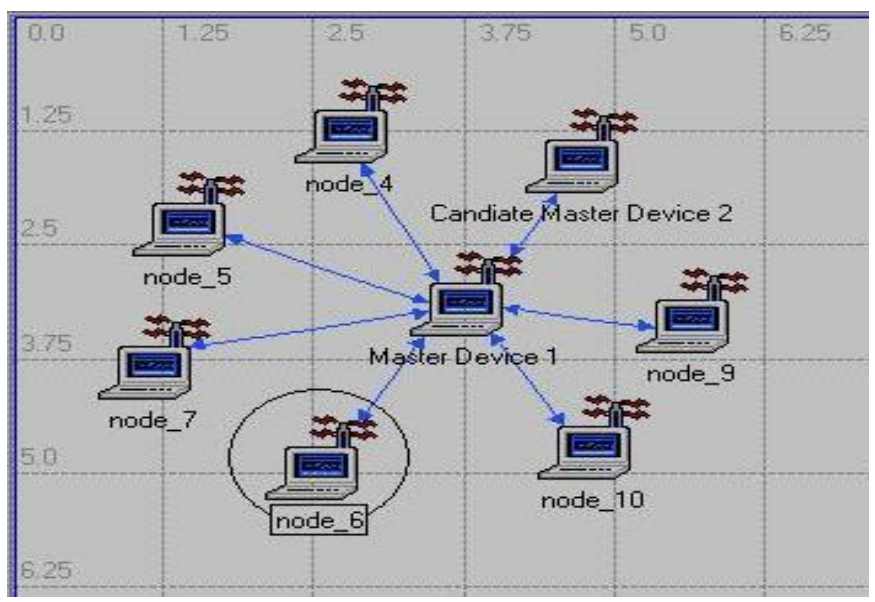

Figure 12. WPAN connect with MD1

Step-4

WPAN 3 now moves to join larger WPAN and now MD1 will

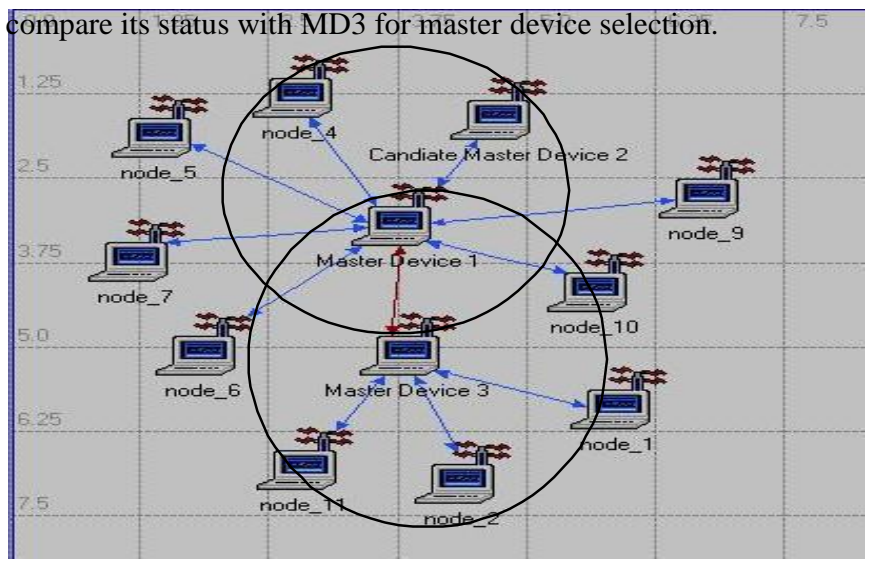

Figure 13. WPAN3 interacting with WPAN1

Step-5

Now MD3 becomes the primary master device and MD1 becomes 
the candidate master device.

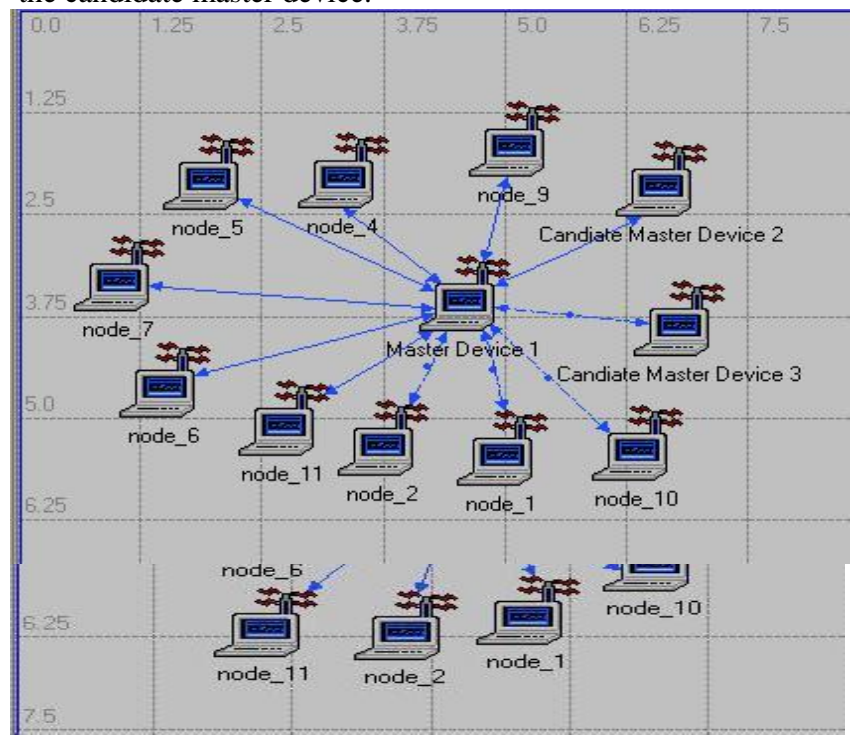

Figure 14. WPAN with two secondary and one primary master device.

\section{Step-6}

Now MD3 went away and the pervious MD1 becomes the primary master device.

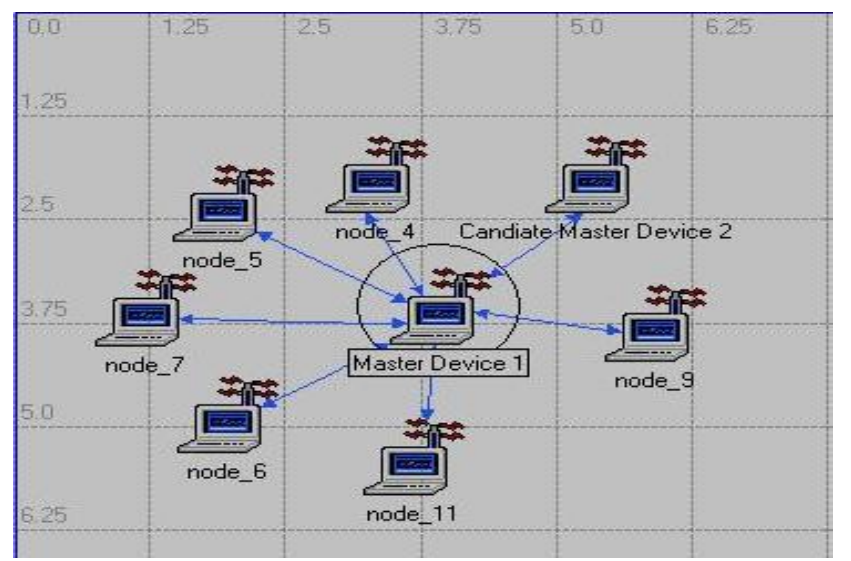

Figure 15. MD1 acts as a primary master device.

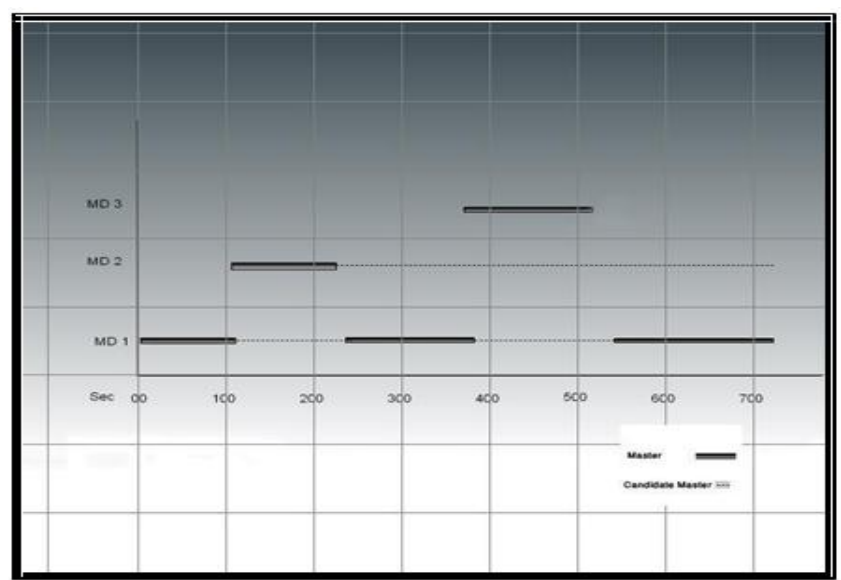

Figure 16. Details of the simulation.
The above graph shows the ping pong effect and the WPAN instability due to the handovers between three master devices.

\subsection{WPAN Stability and Reach ability Results}

This section highlights the discussion on the results of second important factor for master device selection criteria approach used in this thesis. Stability of WPAN devices means the time, which the device spends as a part of WPAN.

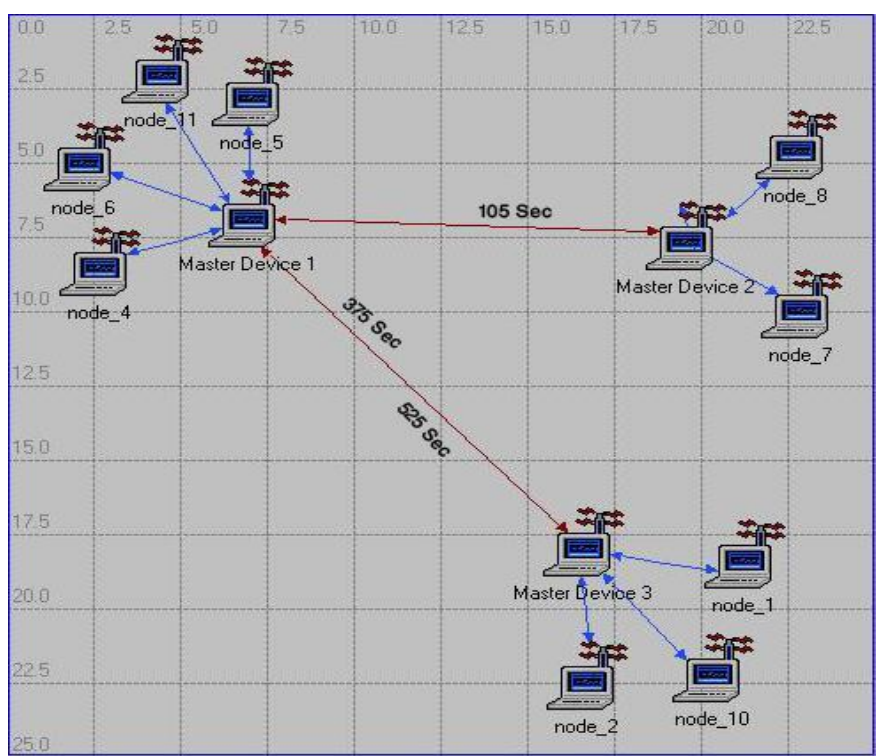

\section{Figure 17. System Model for Simulation}

In this scenario there are three WPANs at a considerable distance and having different number of devices. This scenario will also follows the same sequence as the previous one but this time the master device will be selected according to stability and reach ability parameters. This simulation will also have 2 steps at different timings.

\section{Step1}

When the WPAN1 and WPAN2 interact with each other, the MD1 remains the primary master and MD2 becomes the candidate device. This happens because the stability of MD1 is more than MD2.

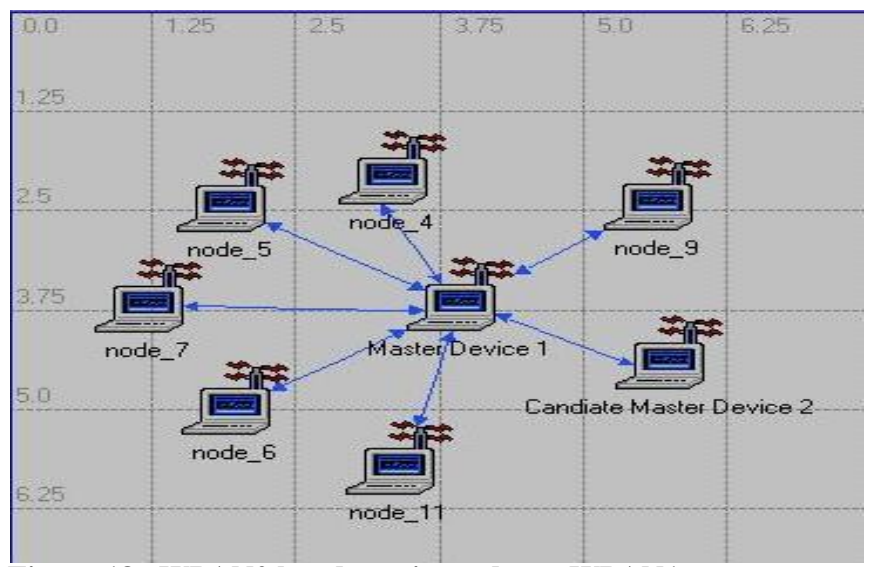

Figure 18. WPAN2 handover its nodes to WPAN1 


\section{Step 2}

WPAN3 also comes in the system boundary of WPAN1 and the MD1 remains the primary master device because of its stability factor.

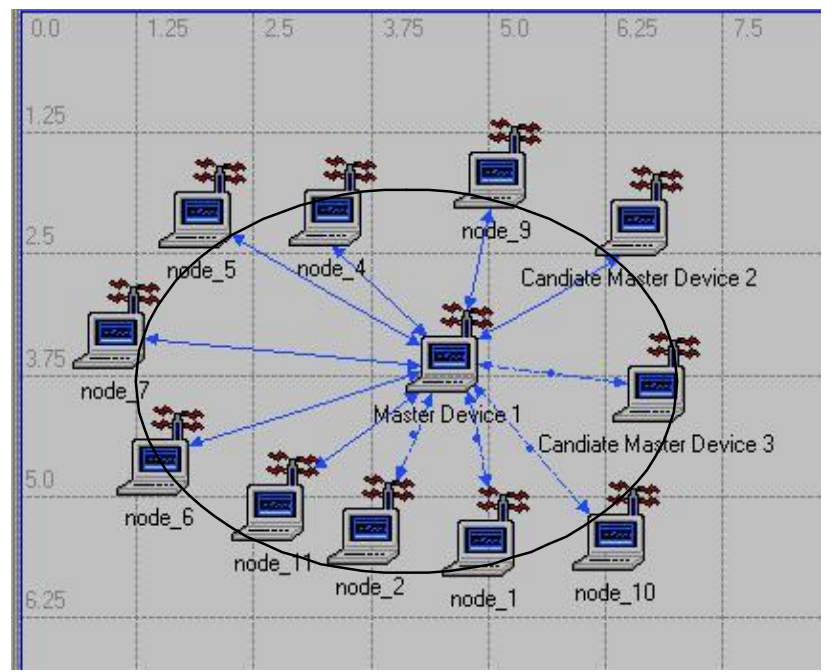

Figure 19. WPAN1 with one primary master device and two candidate master device

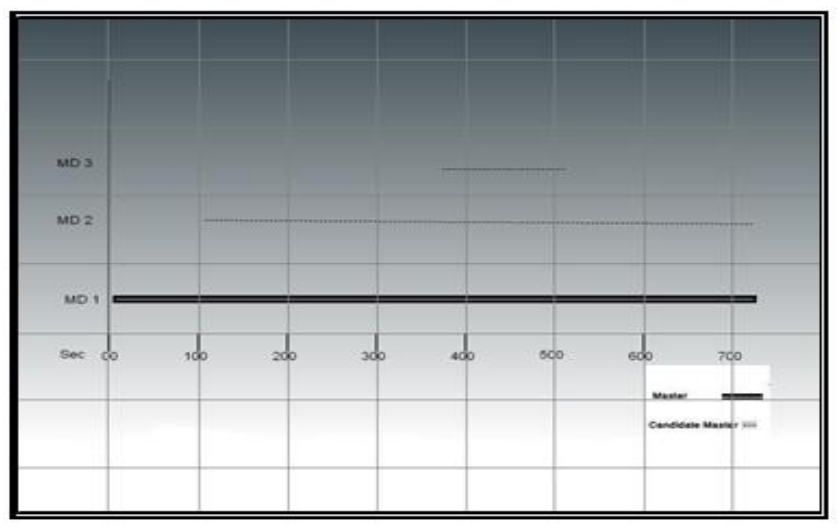

Figure 20. Graphical representation of simulation result

Simulation result in Figure 20. gives a graphical representation, as shown in Figure 17. and Figure 9. Three different WPAN interact with each other and different timing and goes off the network at different timing but due to the selection criteria of the WPAN master device the master device which have more stability in concern of timing remain the primary master device and other master devices becomes secondary master device. The Figure 20. results shows that MD1 remains the master device for 700 sec even when MD2 enters the system at $100 \mathrm{sec}$ and MD3 at 375 $\mathrm{sec}$ of the simulation and leave the system at $525 \mathrm{sec}$ but the system was un-effected. This shows that our propose algorithm provides very stable results and system is also not effect by the ping pong effect.

\section{CONCLUSION}

This research paper reviews the most important factors on master device selection in personal area networks. Here we assumed that Personal Area Network (PAN) behaves in an ad hoc fashion. This paper mainly targets two important parameters in the master device selection for PAN which are Reach ability and Stability. Simulation scenarios present the importance of both criteria. There are other factors for selecting master device, but we focused only on the two main criteria. Simulation results presented in this research to prove that the initiation of a PAN can be started with a TM, which could readily handover to the acquired PAN node which meets the MD selection criteria. We assumed several parameters such as data rate, radio coverage, architecture of PAN and Master/Slave relation. A novel handover technique is introduced for the selection of Master Device. The advantage of this new handover technique is the capability of choosing the Master device in PAN. The results show that the proposed handover technique outperforms other techniques for the selection of Master Device.

\section{ACKNOWLEDGMENTS}

Our thanks to IJCA to give us the opportunity of sharing ideas with human glob and ZTE to give us the practical implementation of the whole work, we thanks our family for their pray and support.

\section{REFERENCES}

[1] Boppana, R.V. Zhi Zheng, "Designing ad hoc networks with limited infrastructure support" Consumer Communications and Networking Conference, 2005. CCNC. 2005 Second IEEE 3-6 Jan. 2005.

[2] Keng Seng Ng, Seah, W.K.G, "Routing security and data confidentiality for mobile ad hoc networks", Vehicular Technology Conference, 2003. VTC 2003-Spring. The 57th IEEE Semiannual Volume 3.

[3] Mehmet-Ali, M.K.; Hayes, J.F, Elhakeem, A.K, "Traffic analysis of a local area network with a star topology"; Communications, IEEE Transactions on , Volume: 36 , Issue: 6 , June 1988.

[4] Prommak, C. Kabara, J. Tipper, D. Charnsripinyo, "Next generation wireless LAN system design", MILCOM 2002. Proceedings Volume 1, 7-10 Oct. 2002.

[5] Tuduce, C. Gross T. "A mobility model based on WLAN traces and its validation", INFOCOM 2005. 24th Annual Joint Conference of the IEEE Computer and Communications Societies. Proceedings IEEE, Volume 1, 1317 March 2005.

[6] Benny Bing, "High-Speed Wireless ATM and LANs", Artech House, UK, 2000

[7] Won Soo Kim, Whan Kim; Seung Eun Hong, Chung $\mathrm{Gu}$ Kang, "A seamless coordinator switching (SCS) scheme for wireless personal area network (WPAN)", Consumer Electronics, 2003. ICCE. 2003 IEEE International Conference on, 17-19 June 2003

[8] G.M.Tamilselvan, Dr.A.Shanmugam Inter and Intra Cluster Scheduling for Performance Analysis of Coexistence Heterogeneous Networks. http://www.ijcaonline.org/archives/ number8/183-319, 2010

[9] Frank Ohrtman and Konrad Roeder, "Building $802.11 \mathrm{~b}$ Wireless Networks", McGraw-Hill @ 2003

[10] Neeli Prasad, Anand Prasad, "WLAN systems and wireless IP for next generation communications" / Artech House, 2002. http://www.networkcomputing.com

[11] T. Bostrom, T. G. Lowe and R. Keller, "Ericsson Mobile Operator WLAN solution", Ericsson Review, No. 1, 2002 . 